\title{
Inhalt / Contents 03/04
}

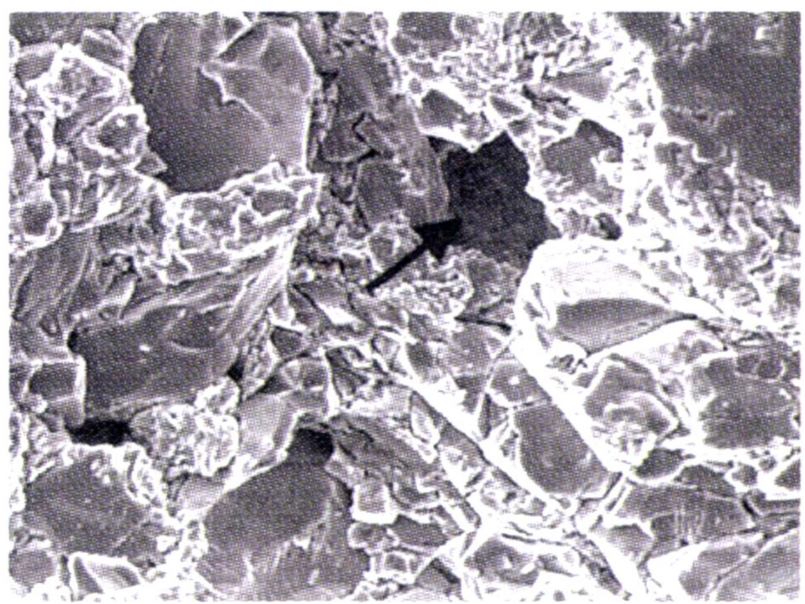

M.-H. Hong

Characterization of Surface Defects in High Strength Galvannealed Steels

Charakterisierung der Oberflächendefekte in hochfesten, nach dem Verzinken wärmebehandelten Stählen

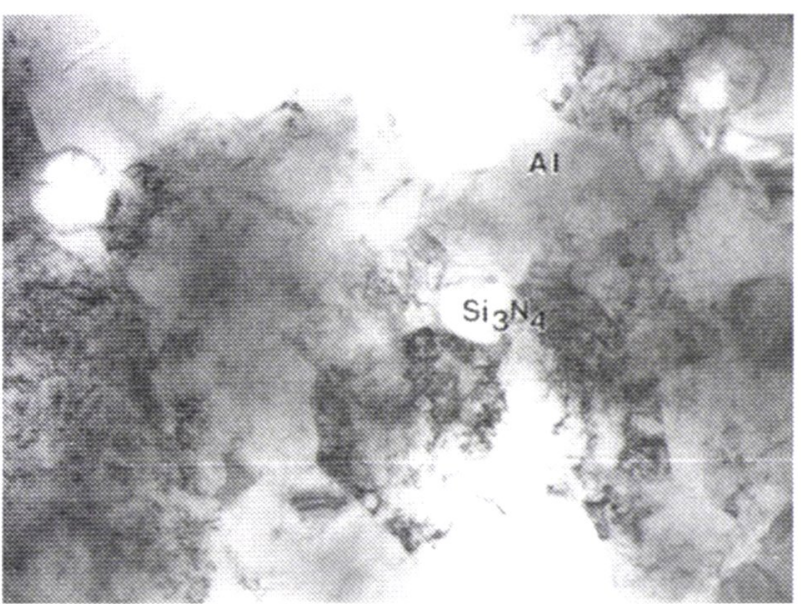

\section{H.-S. Kim, T.-G. Kim, H.-G. Jeong}

Effect of Deformation Temperature and Extrusion Ratio on Microstructure and Superplastic Flow in Aluminum Matrix Composites

Auswirkungen der Verformungstemperatur und des Umformgrades auf Gefüge und superplastisches Fließen in Aluminium-Matrix-Verbundwerkstoffen

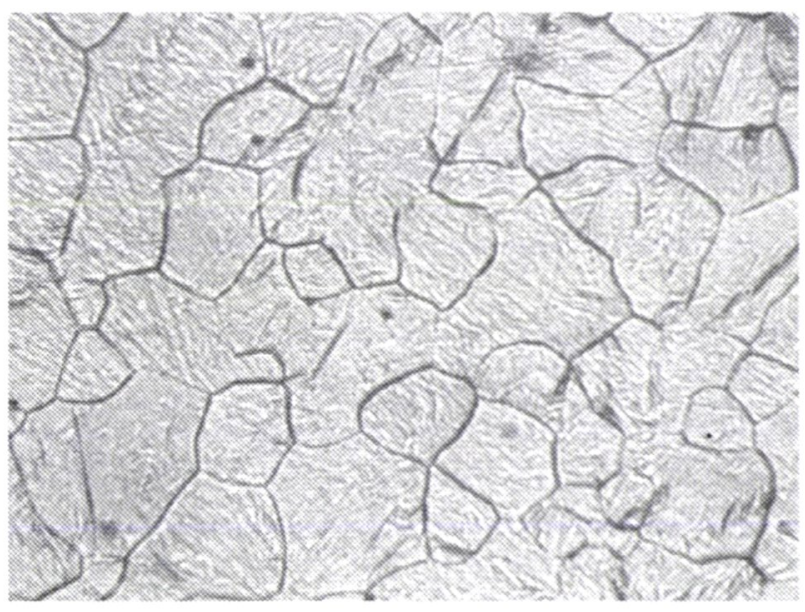

\section{H.K. Kim, C.S. Chung, B.S Cha, B.H. Goo, C.Y. Hyun}

Mechanical Properties and Microstructures of an AZ31 Mg Alloy through Severe Plastic Deformation

Mechanische Eigenschaften und Gefüge einer AZ31-Mg-Legierung nach starker plastischer Verformung

Editorial

Veranstaltungsankündigung/Event Announcement

Literatur-Notizen/Literature Reviews

Mitteilungen/Information

Diskussionsforum/Topic of Discussion 156

Veranstaltungskalender/Meeting Diary 


\section{Praktische Metallographie \\ Practical Metallography}

\section{Gründer/Founders}

Günter Petzow

Gerhard Reinacher +

Charlotte Wachau $†$

\section{Herausgeber/Editor}

Prof. Dr. G. Petzow

\section{Schriftleiter}

Editor in charge

Prof. Dr.-Ing. F. Mücklich

Universität des Saarlandes

Lehrstuhl für Funktionswerkstoffe

Postfach 151150

D-66041 Saarbrücken

Telefon: +49/681 302-2048

Telefax: +49/681 302-4876

E-Mail:pm-editor@matsci.uni-sb.de

\section{Redaktion / Editor}

Dipl.-Ing. S. Mücklich

Zöblitzer Str. 10

D-09125 Chemnitz

Telefon: +49/371/531-5384

Telefax: $+49 / 371 / 531-6179$

E-Mail: silke.muecklich@mb.tuchemnitz.de

\section{Wissenschaftlicher Beirat/Editorial Board}

Dr. C. Bagnall, MCS Associates Inc., Greensburg, PA (USA)

Dr. E. Bischoff, MPI für Metallforschung, Stuttgart

C. Bochert, Buehler GmbH, Düsseldorf

Prof. Dr. H.-E. Bühler, RWTH Aachen

V. DietI, Lette Verein Berlin

Dr. G. Elssner, MPI für Metallforschung, Stuttgart

Prof. Dr. H.E. Exner, TU Darmstadt

Prof. Dr. M. Göken, Friedrich-Alexander-Univ., Erlangen-Nürnberg

Prof. Dr. E. Hornbogen, Ruhr-Universität Bochum

Prof. Dr. F. Jeglitsch, Montanuniversität Leoben

Prof. Dr. C.-K. Kim, KUT, Korea

Dr. H.-J. Klaar, RWTH Aachen

Prof. Dr. A. Kneissl, Montanuniversität Leoben

Dr. W.-U. Kopp, Daisendorf

Prof. Dr. G. Liu, Univ. Beijing, P.R. China

Dr. J. Paul, Leica Vertrieb GmbH, Bensheim

Prof. Dr. M. Pohl, Ruhr-Universität Bochum

Prof. Dr. P.D. Portella, BAM, Berlin

Prof. Dr. Schmitt-Thomas, IST, München

Prof. Dr. G. Schneider, Bosch, Stuttgart

U. Täffner, MPI für Metallforschung, Stuttgart

Dr. J. Trempler, Martin-Luther-Univ. Halle-Wittenberg

Prof. Dr. H.-H. Uchida, Tokai Univ., Hiratsuka, Kanagawa G. Vander Voort, Buehler Ltd., Illinois

Prof. Dr. B. Wielage, TU Chemnitz

Dr. H.-J. Wieland, Verein Dt. Eisenhüttenleute, Düsseldorf

\section{HANSER}

Carl Hanser Verlag, München
Titelseite:

Keramikkondensator im Querschliff

Aufnahme im Hellfeld 50x

(C) Foto: Scott Holt Buehler $\mathrm{GmbH}$

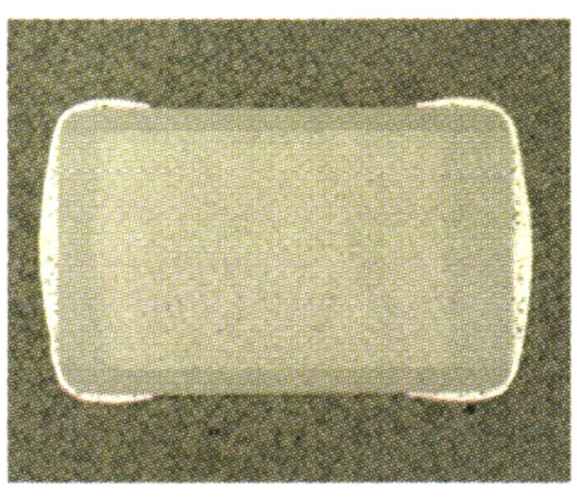

In unserem neu eingerichteten Webshop sind wir rund um die Uhr für Sie da. Besuchen Sie uns: 